\title{
DESIGN OF A WIDE SPECTRAL RANGE AND HIGH- RESOLUTION SPECTROGRAPH FOR MONITORING THE NIGHT SKY BACKGROUND FOR USE IN AIR FLUORESCENCE TELESCOPES
}

\author{
N. ANTONAKAKIS SPYROPOULOS, P. FETFATZIS, \\ E. FOKITIS, V. GIKA, S. MALTEZOS ${ }^{1}$ \\ Physics Department, National Technical University of Athens, Iroon Polytechniou 9 \\ Zografou, Athens, 15780, Greece
}

\begin{abstract}
In this work we present the design of a compact, high-resolution echelle spectrograph for monitoring the Night Sky Background. This spectrograph is combined with a $25.4 \mathrm{~cm}$ telescope and uses an appropriate CCD camera as detector. It can operate in a wide spectral range from Ultraviolet to near Infrared (i.e. $300-780 \mathrm{~nm}$ ) corresponding to the sensitivity spectral window of photocathodes used in the fluorescence telescopes. In the design we also made effort to compromise a sufficient sensitivity and a reasonable exposure time. This instrumentation could become a versatile tool for the operation and development of fluorescence telescopes used in Ultra High Energy Cosmic Ray Experiments.
\end{abstract}

\section{Introduction}

As we have mentioned in the previous ICRC we proceeded to the design and implementation of a dedicated and portable, spectroscopic facility in order to develop a diagnostics and optical noise experimental tool, useful for the statistical analysis of the signal and noise of Night Sky Background Radiation [1]. Our ground based instrumentation approach used existing technology in order to produce a more flexible apparatus that can be adapted by present and planned observatories of UHECRs. In previous measurements of the Night Sky Background Radiation at several locations in Greece we used a low-cost,

\footnotetext{
${ }^{1}$ Corresponding author: maltezos@central.ntua.gr
} 
portable spectrometer based on a concave grating. The results from these measurements were satisfactory although the need for higher spectral resolution and wider spectral coverage in one exposure led us to adapt a more attractive design, based on an echelle grating. This echelle spectrometer is sensitive in the spectral range $300-780 \mathrm{~nm}$, where the photomultiplier detectors typically used in EAS fluorescence installations are sensitive as also the EAS detectors, where the multi-alkali photocathodes have been proposed [2]. In next section, we present the design principles of the echelle spectrometer, while in section 3 preliminary measurements with the already-built prototype echelle spectrometer are discussed. Finally in Section 4, we report the conclusions and prospects for the future work.

\section{Design of the Echelle Spectrometer}

This high-resolution and very compact spectrometer uses an echelle grating with 46.1 grooves $/ \mathrm{mm}$ and $32^{\circ}$ blaze angle as the main dispersing element, while a Fused Silica prism with index of refraction equal to 1.477 at $350 \mathrm{~nm}$ was chosen for the cross-dispersion. Using the equations for an echelle spectrometer that are given in [3] and [4] we choose the most suitable components in order to record the spectral region from $300 \mathrm{~nm}$ to $780 \mathrm{~nm}$ in only one exposure with high resolution and in reasonably small time interval. From these equations the reciprocal linear dispersion (RLD) is about $1.0 \mathrm{~nm} / \mathrm{mm}$ at $350 \mathrm{~nm}$ and the separation between two successive orders is such so the desired 2-D spectrum fills the CCD. A dSLR camera of the SBIG inc. with quite large CCD sensor, 12 $\mathrm{mm} \times 9 \mathrm{~mm}$, and pixel size about $7.4 \mu \mathrm{m} \times 7.4 \mu \mathrm{m}$ is the light detector. According to camera's specifications the quantum efficiency is over $20 \%$ in the

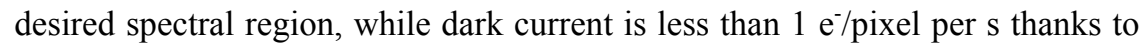
the additional thermo-electrical cooling of the camera. An f/5 UV-grade Fused Silica lens of $250 \mathrm{~mm}$ focal length is used to make the beam parallel before the echelle and a same one to project it on the CCD after its exit from the prism. The wavelength resolution is limited mainly by the $\mathrm{f} /$ number of the $25.4 \mathrm{~cm}$ telescope, which is $\mathrm{f} / 4.7$ and the slit width, which is $75 \mu \mathrm{m}$. Instead of the initial design, which was based in a classic Czerny-Turner mounting, we decide to replace the two mirrors with two UV-grade fused silica lenses. We made this decision for two reasons. Firstly, Fused Silica has very high transmission at the desired wavelengths as can be seen in Figure 1 ( $>90 \%$ from $250 \mathrm{~nm}$ to 1000 $\mathrm{nm}$ ), much higher than the reflectivity of any mirror in that region. Secondly, in the Czerny-Turner mounting each mirror is tilted with respect to its incident chief ray and thus has off-axis aberrations, a problem that is avoided with the 
use of lenses. The only sacrifice for this change is a few centimetres larger spectrometer. In Figure 2 a draw of the spectrometer is shown in respect with the design discussed above.

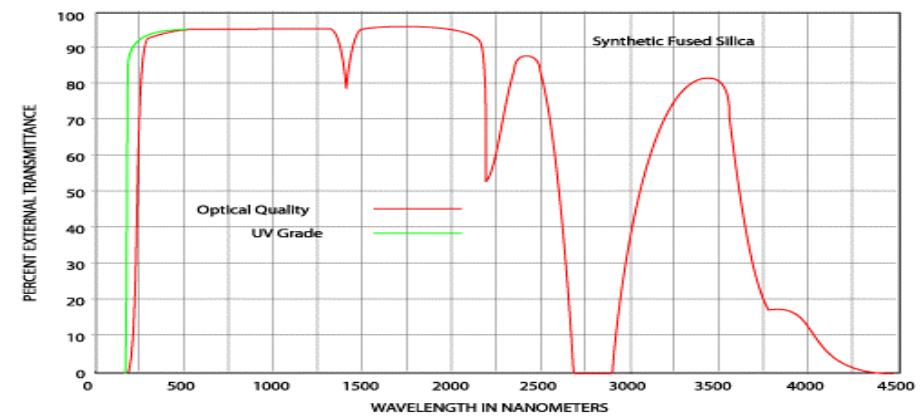

Figure 1. External transmittances for UV-grade and optical grade fused silica.

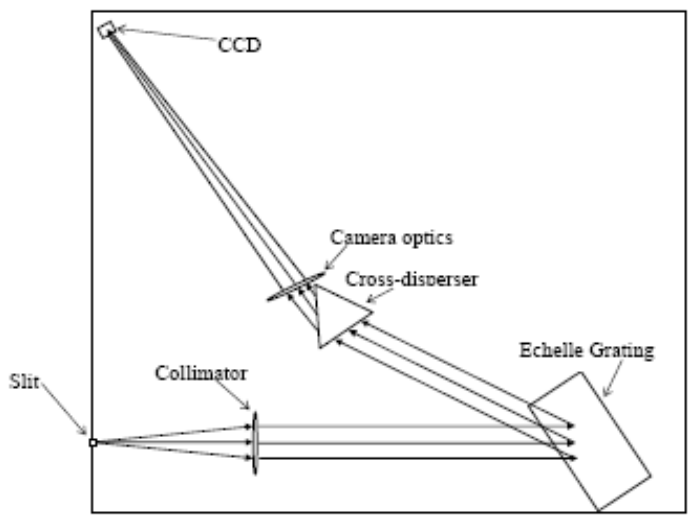

Figure 2. Design of the echelle spectrometer. For more information see the text above.

The choice of the type (diffraction grating or prism) and location (between the echelle and camera optics or between the collimator and the echelle) of the cross-disperser was done according to suggestions from [4] and [5]. The software for analysing the data from the pixels' matrix is IRIS and Visual Spec.

\section{Laboratory Measurements}

We set up a prototype echelle spectrometer for laboratory measurements, consisting of an echelle grating $31.6 \mathrm{~g} / \mathrm{mm}$ and $63^{\circ}$ blaze angle, a dispersing prism made of F2 glass and two lenses of $300 \mathrm{~mm}$ focal length each. For the 
calibration purposes we used an $\mathrm{Hg}$ discharge lamp. As we see in Figure 3, in a single exposure we can record from $400 \mathrm{~nm}$ to $600 \mathrm{~nm}$ while in Figure 4 the FWHM of the $579 \mathrm{~nm}$ line is $0.06 \mathrm{~nm}$ and the reciprocal linear dispersion (RLD) of the $97^{\text {th }}$ order is about $0.5 \mathrm{~nm} / \mathrm{mm}$.

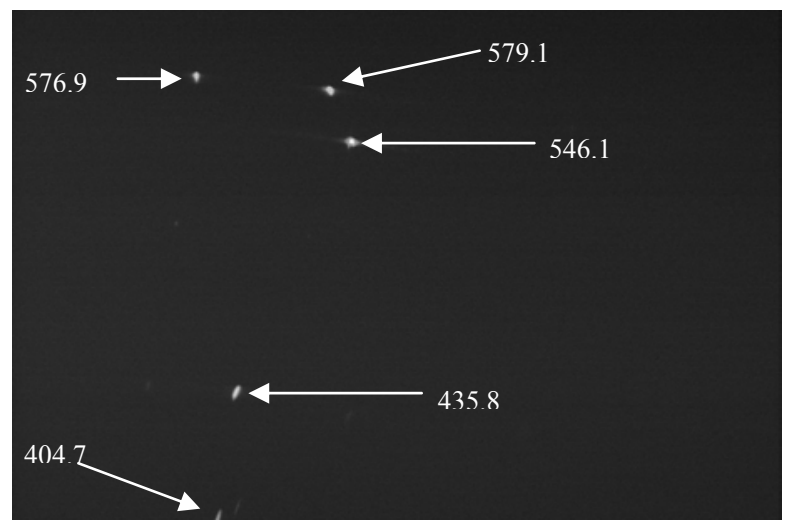

Figure 3. A 2-D picture of $\mathrm{Hg}$ spectrum as recorded from the camera.

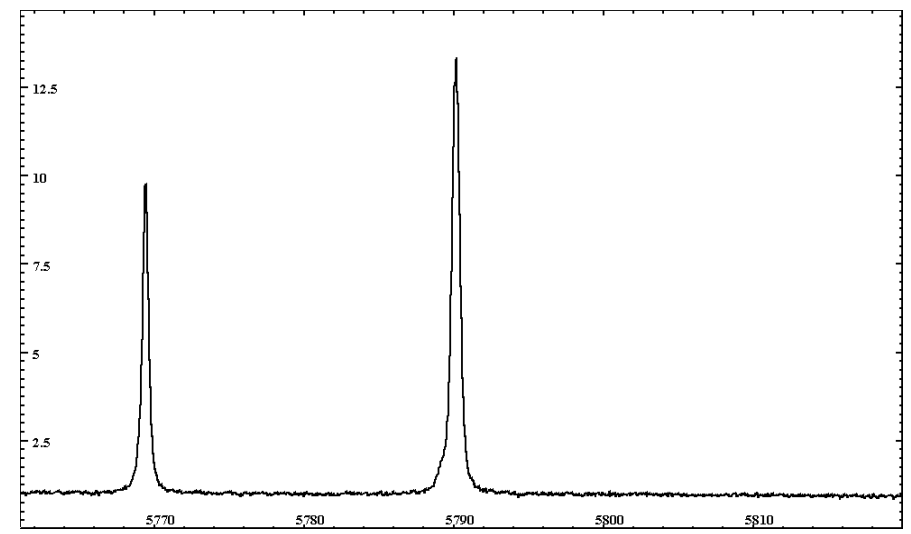

Figure 4 . The spectral analysis of the $97^{\text {th }}$ order where the doublet line $5769 \AA-5791 \AA$ of $\mathrm{Hg}$ appears to be. The $\mathrm{x}$-axis represents the wavelength in $\AA$ and the $\mathrm{y}$-axis the relative intensity.

From these figures it becomes obvious the benefits of an echelle spectrometer by means of resolution and spectral coverage, as mentioned also in [3], [4], [5]. 


\section{Conclusion and Prospects}

In conclusion, we present a high-resolution portable instrument with capability to provide the night sky background in ground based EAS telescope locations. In particular, it can provide the NSB dependence on zenith angle and with further automation, it could give the NSB during nighttime at intervals around 15 min covering the range 300 to $780 \mathrm{~nm}$, which covers the range of sensitivity of bi-alkaly and multi-alkali phototubes. The location where the measurements of the night sky background radiation will be done is situated in the mountain Helmos, near the "Aristarchos" telescope, which is considered as one of the darkest places in Europe. We are planning to insert the collected data in a database, which will be accessible to researchers, developing simulation Codes for EAS phenomena such as CORSIKA, so that spectrally meaningful estimators of night sky background can be developed.

\section{Acknowledgments}

This project is co - funded by the European Social Fund (75\%) and National Resources (25\%) - (EPEAEK II) - PYTHAGORAS. We would like to thank Buil C. author of IRIS and Desnoux V. author of Visual Spec for the free distribution of their software.

\section{References}

1. Fokitis et al. "A system of medium resolution for monitoring the night sky background in the visible and near UV range", in ICRC proc., Merida, Mexico, 2007.

2. G. Garipov et al. "The photomultiplier tubes in the light sensor of an orbital detector for studying the fluorescent radiation of extensive air showers", Instrum. Exp. Tech., 48, 92-95, 2005.

3. D. Schroeder. "Design Considerations for Astronomical Echelle Spectrographs", PASP, 82, 1253, 1970.

4. D. Schroeder. "Astronomical Optics", Academic Press San Diego, $2000^{2}$.

5. D. Walker and F. Deigo. "Design Philosophy of the Forthcoming Echelle Spectrographs for the AAT and LPO", Mon. Not. R. Astron. Soc. 217, 355, 1985. 